Instituto Internacional de Investigación y Desarrollo Tecnológico Educativo INDTEC, C.A.

DOI: https://doi.org/10.29394/Scientific.issn.2542-2987.2019.4.11.8.156-176

OAI-PMH: http://www.indteca.com/ojs/index.php/Revista Scientific/oai

Artículo Original / Original Article

\title{
La Transcomplejidad como Agente Integrador de las Tecnologías de Información y Comunicación
}

\author{
Autora: Mayibe Josefina Agüero Rodríguez \\ Universidad Centroccidental "Lisandro Alvarado", UCLA \\ mayibe.j.aguero@gmail.com \\ Barquisimeto, Venezuela \\ https://orcid.org/0000-0003-4101-2166
}

Resumen

La presente investigación de carácter cualitativo, se abordó mediante el método fenomenológico hermenéutico. El objetivo es conocer la visión que sobre la transcomplejidad como agente integrador de las tecnologías de información y comunicación, posee los docentes universitarios del Decanato de Ciencia y Tecnología de la (UCLA). La metodología se centró en la recopilación de información mediante la técnica de entrevistas en profundidad. En relación con los informantes claves fueron tres (3) docentes adscritos al Departamento de Estudios Básicos y Sociales llamados versionantes. Se tiene como propósito que el educador incorpore nuevas estrategias didácticas para innovar, en otros términos, conocer para crear nuevos conocimientos, más allá del saber técnico-aplicacionista, además de responder a un planteamiento centrado en proponer lineamientos que permita un manejo reflexivo desde una mirada compleja y transdisciplinar. Una vez develada la realidad, a partir de los versionantes y contrastada con los referentes teóricos, concluyo que los docentes universitarios deben integrar las innovaciones tecnológicas al proceso enseñanza y aprendizaje, porque no puede haber innovación en la educación universitaria, sin las innovaciones tecnológica y nuestras universidades son las que deben propiciar un encuentro del docente universitario ante las innovaciones tecnológicas, que garanticen su adaptación a las necesidades de la sociedad actual.

Palabras clave: educación; aprendizaje; enseñanza superior; tecnología de la información.

Fecha de Recepción: 19-05-2018

Fecha de Aceptación: 28-08-2018 


\title{
Transcomplexity as an Integrating Agent of Information and Communication Technologies
}

\begin{abstract}
The present investigation of qualitative character, was approached by the hermeneutical phenomenological method. The objective is to know the vision that on the transcomplexity as an integrating agent of information and communication technologies, has the university professors of the Dean of Science and Technology of the (UCLA). The methodology focused on the collection of information through the technique of in-depth interviews. In relation to the key informants were three (3) teachers assigned to the Department of Basic and Social Studies called versionantes. The purpose is that the educator incorporates new didactic strategies to innovate, in other words, to know to create new knowledge, beyond the technical-applicationist knowledge, in addition to responding to an approach focused on proposing guidelines that allow reflective management from a perspective complex and transdisciplinary. Once the reality is revealed, starting from the versions and contrasted with the theoretical referents, I conclude that university teachers must integrate technological innovations into the teaching and learning process, because there can be no innovation in university education, without technological innovations and our Universities are those that must promote a meeting of the university professor before the technological innovations, that guarantee their adaptation to the needs of the current society.
\end{abstract}

Keywords: education; learning; higher education; information technology.

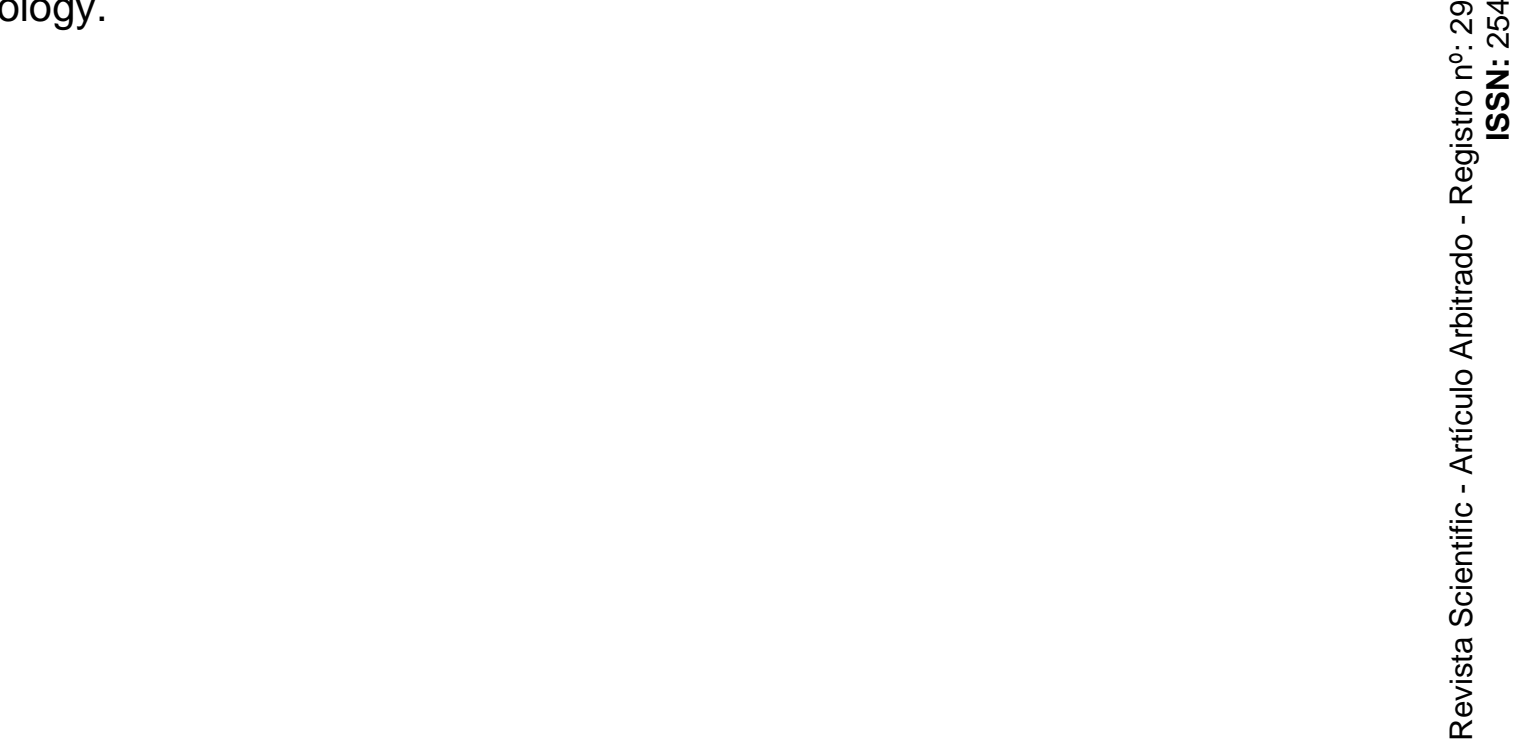

Date Received: 19-05-2018

Date Acceptance: 28-08-2018 


\section{A manera de Introducción}

Las fluctuaciones en el contexto mundial, tomados en consideración científicamente bajo el paradigma posmoderno, proporcionan variaciones en todas las aristas del quehacer del hombre, y en consecuencia, en el sector educativo. No encontramos a nivel educativo con un cambio de paradigma del viejo modelo a uno más humanista, con características más enmarcadas en el plano subjetivo y humano, sumado a la rapidez de las variaciones en el medio circundante.

Desde mi perspectiva, mantener en un equilibrio el nivel de enseñanza universitaria en relación con la de los estudiantes, obliga a la planta de docentes a mantenerse en una constante formación y actualización, que les permita entender y gerenciar las demandas de la sociedad del conocimiento tanto extra como intra curricular con el fin de fomentar su creatividad, competencias y talentos dando respuestas a las exigencias del entorno.

En tal sentido, este fenómeno en desarrollo reta a las casas de estudios universitarios, específicamente a las venezolanas a incorporar a sus comunidades bajo un enfoque científico-humanista donde el proceso del enseñaje requiera de su participación, enmarcado en los esquemas y procesos adecuados y exigidos por las normativas vigentes.

Sin embargo, la dificultad preponderante a la que nos enfrentamos actualmente, es la de ubicar la educación desde un enfoque diferente a la que siempre se ha estado acostumbrado, es decir, un cambio no de forma sino de estilo, tal como lo plantea Morín (2010): cuando dice que es “... una reforma no programática, sino paradigmática, que concierne a nuestra aptitud para organizar el conocimiento" (pág. 22); por lo que puedo señalar que, conocer la esencia del hombre no implica extraerlo del mundo en que vive, se trata más bien de darle su ubicación en él.

Desde esta concepción, la educación como espacio de saberes, no sólo debe estar centrada en los paradigmas de pensamiento, en la investigación y 
en la generación de teorías o modelos, sino en buscar vías operacionales que procuren modelar y transformar la praxis educativa en todos sus niveles, claro está, dejando a un lado la visión reduccionista y lineal de la ciencia.

Bajo los cánones de esta perspectiva, el presente estudio no busca solo dar explicaciones sino comprender, interpretar y reflexionar desde una mirada transcompleja en un contexto complejo y transdisciplinario, lo que está más allá de las disciplinas en espacios de saberes, que según González (2012): "es buscar lo que está entre, a través y más allá de las disciplinas mismas. Visto en términos educativos, se trata de una nueva forma de vivir y convivir en la humanidad" (pág. 8); esto fortalece la conceptualización del presente estudio, ya que tiene como finalidad plasmar lineamiento en busca de un aprendizaje más reflexivo, dinámico dando paso a la construcción y reconstrucción en el proceso del enseñaje.

Es pertinente resaltar que, el incorporar las Tecnologías de la Información y Comunicación (TIC), a manera de agilizar el proceso del aprendizaje nos conduce a realzar su valor, a la vez de reflexionar su eficacia en la enseñanza. La didáctica en la actualidad es quien se encarga de organizar las reglas de la metodología a utilizar para que el aprendizaje fluya eficientemente. Tal vez este sea el motivo que nos conduzca a comprender el "como" de la enseñanza, la cual ha llevado al solapamiento de ésta con el factor metodológico, una característica primordial de la didáctica que ha sido olvidada en los últimos años.

En tal sentido, es a partir de allí que formamos parte de un conglomerado social que está sufriendo cambios por los inminentes avances tecnológicos, como lo son las redes sociales, la internet, la televisión por cable y satelital, entre otros, en función de los beneficios que estos nos ofrecen de manera acelerada e impresionante. Es necesario acotar que, las (TIC's) han tenido un avance impresionante en estos últimos años, es tanto así, que le has dado paso a lo que hoy día se conoce como Sociedad del Conocimiento o de 
la Información, además, que no hay sitio, lugar o espacios del ser humano que estén aislados al impacto de este desarrollo.

En otro orden de ideas, al estudiar la transcomplejidad como agente integrador de las TIC desde una visión del docente del Decanato de Ciencia y Tecnología (DCyT) de la (UCLA), es necesario conocer los entornos en los cuales se desenvuelven los educadores de la institución objeto de estudio, además de ellos como tal; por su parte, González (2007): cita que "la subjetividad está constituida tanto en el sujeto individual como en los diferentes espacios sociales en que este se desarrolla" (pág. 17); esto motivado a la necesidad de observar el entorno de estudio desde una visión poliocular.

En sintonía con esta misma línea, la incorporación de las TIC en la praxis educativa de los docentes de la (UCLA) perteneciente al (DCyT), la investigadora pretende permear una escenario plegado de múltiples posibilidades y de inconmensurables retos, donde se busque avanzar hacia una comprensión y una acción más profunda, completa y dialéctica sobre la realidad cambiante, abriendo espacios para la emergencia de posturas heurísticas y creativas que intenten modelar pensamientos, sin pretender convertirse en nuevos dogmas, sino que constituyan otra manera de repensar en escenarios complejos y múltiples, no de forma aislada, unívoca, reductiva y excluyente.

Ahora bien, con la finalidad de modelar dicho pensamientos en el docente, es inevitable el fortalecimiento de los procesos cognitivos y de enseñanza, motivado a que es imposible abordar problemas complejos desde una sola arista o disciplina, para ello, se debe desarrollar la pericia del docente, su sincretismo, que le permita la construcción en colectivo de nuevas herramientas, estrategias, recursos, entre otros y así enfrentar nuevos escenarios, en fin nuevos conocimientos transdisciplinarios y complejos, como respuestas a las emergentes demandas educativas de la sociedad postmoderna. 
Desde las consideraciones anteriores, es muy importante resaltar que el uso de las (TIC`s) por parte del docente universitario, como una herramienta didáctica en sus procesos didácticos se han traducido como una carencia de seguridad, motivación, miedo, desinterés, pasividad, y sobre todo de reflexión acerca de la forma en la que deben incorporarse y los posibles cambios que generarían en el resto de los elementos del acto de la enseñanza.

De lo indicado, la investigadora señala de manera clara y concreta, que el educador debe manejar un abanico de estrategias innovadoras que le permita a sus educandos involucrarse de manera efectiva en cualquier actividad propuesta, haciendo uso de la investigación y los recursos tecnológico disponibles actualmente en el proceso de enseñanza y aprendizaje, ya que el docente no solo debe basarse en el desarrollo de su praxis pedagógica, impartiendo contenido o información que desvíe la atención de sus discentes sino que contribuya a la producción de un aprendizaje significativo.

Es de acotar, que la autora del presente estudio en la búsqueda de contribuir a la mejora de la academia, realizó la investigación bajo el enfoque cualitativo, con apoyo del método fenomenológico hermenéutico y la metodología se centró en la recopilación de información mediante la técnica de entrevista en profundidad.

El propósito de este artículo es conocer la visión que sobre la transcomplejidad como agente integrador de las tecnologías de información y comunicación, posee el sistema universitario del Decanato de Ciencias y Tecnologías de la (UCLA), considerando que, un educador es capaz de poseer habilidades y destrezas profesionales y que difícilmente puede desarrollarse si previamente no consigue un dominio de su competencia. Ante lo planteado, la pregunta es ¿Cuál sería la visión que tienen los docentes universitarios del (DCyT) de la (UCLA) en cuanto a la integración de las (TIC`s) bajo la transcomplejidad? 
Dentro de este contexto, surge para la investigadora el siguiente propósito: Conocer la visión que sobre la transcomplejidad como agente integrador de las tecnologías de información y comunicación, posee el sistema universitario del Decanato de Ciencia y Tecnología de la Universidad Centroccidental Lisando Alvarado de Venezuela.

\section{Referentes Teóricos}

\subsection{Antecedentes}

He tomado como antecedente, por brindar un apoyo a mi artículo el cual busca la incorporación de las (TIC`s) bajo una visión transcompleja en la praxis del docente universitario, la tesis doctoral presentada en la Universidad de Jaén, Andalucía España, por Morales (2014a), titulada:

Percepción del profesorado y del alumnado de la facultad de ciencias de la educación de su universidad de Granada, acerca de la utilización de las TIC por parte del profesorado universitario y de su integración en el proceso de enseñanzaaprendizaje (pág. 1).

Dicha tesis tiene como objetivo, según Morales (2014b): "Describir la opinión y percepción del profesorado universitario acerca de la utilización de las TIC y la integración de estas en sus procesos de enseñanza", (pág. 203). La cual pretende conocer en relación con la utilización de las TIC en la educación superior, tal como lo señala Morales (2014c): "la valoración de los profesores universitarios sobre la integración de estas en sus procesos de enseñanza" (pág. 199).

Entre las conclusiones presentadas por la autora, he tomado la más significativa para el desarrollo de mi estudio la cual plantea Morales (2014d): cuando afirma que, "el profesorado considera que es necesario un proceso de cambio y adaptación para que la introducción de las TIC sea plena por parte del profesorado" (pág. 433); de allí que, estos cambios deben estar 
enmarcados en las exigencias de la sociedad para permitirle al docente adaptarse al cambio, de manera que sea necesaria una formación en competencias para el dominio de las (TIC) en los procesos de enseñanza aprendizaje.

La vinculación del trabajo de Morales con el presente estudio, es la necesidad de dotar a los docentes de herramientas y estrategias innovadoras para ser incorporada en el desarrollo de sus procesos diarios de enseñanzas y aprendizajes, a fin de lograr que sus discentes obtengan un aprendizaje significativo, haciendo uso de técnicas relacionadas directamente con las (TIC`s), donde se pueda plasmar diferentes medios didácticos.

\subsection{Referentes Teóricos}

Este apartado tiene como finalidad presentar la fundamentación teórica del artículo de investigación, el cual tiene como propósito Conocer la visión que sobre la transcomplejidad como agente integrador de las tecnologías de información y comunicación, posee el sistema universitario del Decanato de Ciencia y Tecnología de la Universidad Centroccidental Lisando Alvarado, Venezuela. Además, se centra en los siguientes aspectos: Transcomplejidad, (TIC`s), Didáctica Universitaria.

Desde esta perspectiva, existen muchas formas de ver e interpretar la realidad del mundo, dependiendo del punto de vista, las vivencias, las experiencias, las creencias de cada persona, estás a su vez se relacionan con una visión epistemológica transdisciplinaria y compleja, que reacciona ante los patrones cognitivos establecidos, bien sea por herencia o por uso.

\subsubsection{Educación Transcompleja "Más allá de la Complejidad"}

La educación es la base fundamental en cualquier proceso de transformación del individuo en la sociedad, es la fórmula precisa de cambios complejos y de aplicación de estrategias de investigación transdisciplinar. 
Desde esta visión, la educación, junto al trabajo, constituye uno de los medios primarios para obtener una mejor calidad de vida en nuestro entorno que debe ser vista como un todo, pero a su vez parte de un mundo representado por la complejidad, por invariables cambios, la incertidumbre, lo caótico, el cual no puede ser descubierto, ni su problema resuelto desde la óptica del pensamiento clásico que ha sostenido la ciencia hasta nuestros días.

Esta teoría sobre la educación transcompleja propone una transformación del sistema educativo, que considere esencialmente la aplicación en un contexto complejo y transdisciplinar, es decir, proponer lineamientos que permitan responder al reduccionismo, aplicado desde su origen al nivel de educación primaria venezolana, donde se enseña apartar los objetos de su entorno, a separar las disciplinas más que a reconocer sus apoyos, a desunir los problemas más que a vincularlos e integrarlos.

Por tal motivo, la autora del presente artículo enfatiza sobre la necesidad de indagar otros modos y formas de transmitir conocimientos, que permitan asumir la educación desde diversos escenarios, de forma integral, de manera compleja y no desde una sola arista o de manera disciplinar, para ello, se debe tomar en cuenta tres elementos como lo son la pedagogía, el currículo y la didáctica.

Es oportuno señalar, que si se desea una transformación en la educación universitaria, no puede seguir funcionando en la actualidad desde una visión disciplinaria y simplista. Por ende, la autora Villegas y Schavino (2012): reflexiona acerca de "la educación desde una visión transcompleja, la cual es asumida como una nueva cosmovisión de complementariedad, donde múltiples visiones se integran para construir propuestas pedagógicas, didácticas y curriculares que fortalezcan la posibilidad de desarrollar seres autónomos" (pág. 55); es decir, que sean libres de pensamientos, reflexivos, creativos, críticos, que puedan establecer mediante diálogos de saberes, reciprocidades argumentativas y propositivos de aportes y no de imposiciones. 
Por esta razón, la investigadora destaca que para incorporar las (TIC) en el proceso de enseñanza-aprendizaje, todos los actores del sector educativo a nivel superior debe tener presente estos tres elementos desde una base transcompleja, con el fin de reforzar el modelo académico como fortaleza y proporcionando un clima motivador, que en concordancia con el componente tecnológico, deje a un lado las prácticas tradicionales que dan muestra de agotamiento y decadencia en el logro de las actividades, sino asumir una forma novedosa de hacer las cosas.

\subsubsection{Las TIC's como Agente Integrador desde la Transcomplejidad}

Las Tecnologías de Información y Comunicación (TIC`s), desde mi perspectiva avalada con mi experiencia profesional y docente, consisten en una serie de herramientas que manipulan información, ya sea por medio de redes de computadoras, redes sociales, medios escritos e impresos, radio y televisión, que para el caso de mi investigación, la información es de carácter educativo y la misma pueda llegar a cualquier ámbito de la sociedad. La importancia de estas herramientas es que adicionalmente constituyen estrategias innovadoras que facilitan el registrar, almacenar y difundir contenidos informacionales en la web, como lo es el caso de los blogs, el podcast y por supuesto las redes sociales.

Las (TIC`s) son medios y no fines para todo tipo de aplicaciones en el sector educativo, es decir, son herramientas que ayudan a facilitar el proceso de aprendizaje, la adquisición de habilidades y abre al camino a diferentes formas de aprender, amoldándose a los diferentes estilos y ritmos de los participantes. De modo que la internet deja de ser un instrumento especializado de uso solo de la comunidad científica a ser un repositorio de información que se encuentra al alcance de cualquier persona.

El sistema educativo a nivel mundial y en particular, los países latinoamericanos afrontan el reto de incorporar las (TIC's) en su sistema 
educativo, para que sus estudiantes se apoderen de herramientas innovadoras que les serán de utilidad en la actualidad. Con la arribada de las tecnologías, el docente se ha visto en la necesidad de incursionar sus prácticas hacia una formación centrada en el educando, dejando de un lado el pizarrón y el discurso, enfocándose más hacia clases magistrales y convirtiéndose en un facilitador de enseñanza, a pesar de la apatía, pasividad que todavía existe en las aulas.

Por consiguiente, para poder disfrutar de estos avances tecnológicos, se requiere formar y capacitar al profesional de la educación en el manejo de recursos tecnológicos, como lo son computadoras, Tablet, celulares inteligentes, redes sociales, software, entre otros. Este nuevo evento social implica un esfuerzo y un cambio paradigmático que emerge de una disrupción para adaptarse a una nueva forma de vida, donde se tendría la oportunidad de incorporar en forma integral a las personas por medio de estrategias educativas e innovadoras acordes con nuestro siglo.

El empleo de las (TIC`s) desde un enfoque integrador coadyuvan a la sustitución de métodos obsoletos, por métodos integradores, en el cual se busca cambiar que el estudiante deje de ser pasivo, estático y solo receptor de conocimientos y se convierta en un aprendiz participativo y dinámico. También da la oportunidad de integrar un triángulo interactivo entre docentecontenido-objetivos de aprendizaje-estudiantes, representando un papel esencial en el proceso del enseñaje.

\subsubsection{El Sector Universitario en el Contexto de la Transcomplejidad}

Es importante contextualizar la universidad, por ser un elemento clave por su articulación directa con el entorno, donde se llevará a cabo el desarrollo de la futura generación de profesionales de nuestro país. La universidad según el Diccionario de la lengua española, editado por la Real Academia Española, DRAE (2017): "es una institución de enseñanza superior que comprende 
diversas facultades, y que confiere los grados académicos correspondientes.

Según las épocas y países puede comprender colegios, institutos, departamentos, centro de investigación, escuelas profesionales, entre otras" (párr. 1).

Al referirnos a las universidades venezolanas, según la Ley de Universidades (1970): en su artículo 1, expresa que "es una comunidad de intereses espirituales que reúne a profesores y estudiantes en la tarea de buscar la verdad y afianzar los valores trascendentales del hombre" (pág. 1). Desde este enfoque, el conocimiento siempre anda en busca de la verdad, sin obviar los valores humanos, se puede determinar su aceptación o no.

Es necesario acotar que, nuestras universidades se encuentran en desventajas respecto a los países desarrollados, Albornoz (2006): afirma que "el papel de la educación superior en nuestros países, sobre todo en las Universidades, es el de asumir el papel de intérpretes de aquello que acontece en el mundo y traducirlo a nuestras dimensiones y necesidades..." (pág. 28). En vista de que, no se puede competir con los gigantes en estas complejas áreas del conocimiento, pero si, podemos montarnos sobre sus hombros y avizorar el porvenir.

En sintonía con lo señalado y como cierre de este apartado, considero que la educación universitaria como vía para afianzar el progreso y asegurar el desarrollo del país, por ser su objetivo primordial el forjar y crear conocimientos, es un factor esencial en el crecimiento social, pero la actual demanda en decremento y la situación del docente han llevado a un desequilibrio en la formación del talento humano.

Teniendo en consideración, la diversidad de problemas que en la actualidad están presentando las universidades venezolanas, la (UCLA) no escapa de esta realidad, ya que en estos momentos arrastramos un déficit financiero que imposibilita la construcción de nuevas edificaciones o mantenimiento y adecuación de las que ya existen, en vista de que no se 
pueden adquirir tecnologías de puntas, problema en el que no está inmersa solo la universidad sino la población en general, de tal modo que es imposible hacer frente a la actual revolución tecnológica. Claro está, que aunque se presentan conflictos, los cambios deben iniciarse desde el componente humano, quien al final será el beneficiado real.

\section{Plano Metodológico}

Este apartado está dirigido a revelar la forma en la cual se desarrolló el proceso de la investigación y el significado del conocimiento, teniendo como propósito conocer la visión que sobre la transcomplejidad como agente integrador de las tecnologías de la Información y Comunicación, poseen el sistema universitario del Decanato de Ciencias y Tecnología. Se pretende abordar el estudio desde la metodología cualitativa que, según Sandín (2003): es aquella "orientada a la comprensión en profundidad de fenómenos educativos y sociales, a la transformación de prácticas y escenarios socioeducativos, a la toma de decisiones y también hacia el descubrimiento y desarrollo de un cuerpo organizado de conocimientos" (pág. 123).

\subsection{Sustento Metodológico - Método}

En coherencia a lo planteado se abordó el fenómeno bajo el enfoque cualitativo, sobre este particular, Taylor y Bogdan (2002), consideran que es una "investigación que produce datos descriptivos: las propias palabras de las personas, habladas o escritas y la conducta observable" (pág. 20); esto se debe a que un estudio cualitativo persigue la compresión del fenómeno en estudio en su entorno natural. Adicionalmente, Molina (2018): plantea que "desde la perspectiva del paradigma cualitativo, es inaceptable desligar pensamiento y realidad, se tiene la convicción sobre la realidad modelada y construida por los pensamientos" (pág. 131).

Desde este matiz, se puede aseverar que la presente investigación es 
cualitativa, debido a que busca una relación directa con el objeto de estudio donde su participación es subjetiva, y su propósito es conocer la visión que sobre la transcomplejidad como agente integrador de las tecnologías de información y comunicación, posee el sistema universitario del Decanato de Ciencias y Tecnologías de la (UCLA).

Para lograr la integración de las (TIC`s) desde el escenario de la transcomplejidad, se requiere accionar en el ámbito del objeto de estudio bajos escenarios complejos e innovadores; con el fin de contribuir ofreciendo un bagaje de herramientas, que pueden ser incorporadas en cualquier momento, con el fin de favorecer su praxis educativa.

\subsubsection{Método}

Si bien es cierto, que la encargada de enlazar el sujeto con el objeto es la metodología y sin ella sería imposible llegar al conocimiento científico; cabe señalar, que quien lleva a demostrar la validez de los argumentos de manera más sencilla es el método. De allí, que el autor Gadamer (1996): manifieste que "el método se constituye en una ayuda a la estrategia de investigación, lo cual permite convertirla en un proceso creativo, abierto y flexible a los diálogos y a la forma de expresión que favorecen a las comunicaciones intersubjetivas" (pág. 23).

En virtud de los aspectos mencionados, el presente estudio fue abordado mediante el método fenomenológico, según Martínez (2004): “El método fenomenológico se centra en el estudio de esas realidades vivenciales que son poco comunicables, pero que son determinantes para la comprensión de la vida psíquica de cada persona" (pág. 139); ya que trata de comprender las vivencias desde una visión particular y colectiva para así alcanzar a develar la realidad que se han presentado, manteniendo conciencia del hecho.

Por otro lado, mi percepción como investigadora del estudio me permitió develar el significado de las palabras de mis versionantes, es de allí que, 
expreso que es hermenéutica por tratarse de un proceso interpretativo de vivencias, tratando de comprender las situaciones o realidades en cualquiera de sus manifestaciones, esto es afianzado por Habermas (1986), citado en Sosa (2015): quien manifiesta que "la hermenéutica como condición trascendental de la interpretación-comprensión, es una forma penetrante de discernimiento con el principio racional” (pág. 16).

\subsubsection{Técnicas e Instrumento de Recolección de la Información}

En lo referente a las técnicas utilizadas para recolectar la información de los versionantes claves seleccionados para el desarrollo del presente estudio se utilizó la entrevista en profundidad, realizada de tal manera que los versionantes comunicaron libremente sus vivencias, experiencias como docentes formadores en el proceso del enseñaje. Las preguntas y respuestas producto del diálogo entrevistador-entrevistado, las grabé y posteriormente las transcribí para su comprensión, interpretación, codificación y categorización, respetando las expresiones tal como fueron concebidas.

En cuanto al análisis e interpretación realizado, con apoyo en la hermeneusis, desentrañe las realidades de cada uno de los versionantes mediante sus experiencias y vivencias develadas en las entrevistas, para lo cual, se interpretó el significado por categorías emergentes, apoyadas por frases textuales de los versionantes. Obteniéndose los primeros hallazgos de la triangulación por categorías de lo expresado por cada uno de los versionantes.

\section{Presentación de los Hallazgos}

Esta realidad obtenida, me permitió conocer e interpretar desde la transcomplejidad, como se integran las TIC's en el sistema universitario, además desde las voces de los versionantes obtenidas en las entrevistas realizadas, tabuladas y de las cuales se presentan los extractos que dan origen 
a las categorías Déficit Presupuestario y TIC's. (Cuadro nro. 1).

Cuadro nro. 1: Extractos Categorización Versionantes.

\begin{tabular}{|c|c|}
\hline Texto & Categoría \\
\hline $\begin{array}{l}\text { Interrogante 1: ¿Cuáles estrategias didácticas } \\
\text { utilizas para desarrollar tus actividades en el aula? } \\
\text { Extracto del Versionante 1: } \\
\text {...gusta la tecnología, dentro de los salones de la UCLA } \\
\text { contamos con recursos tecnológicos que a pasar de ser } \\
\text { obsoletos nos pueden auxiliar en estos casos, además, } \\
\text { existe conexión a internet y aunque muy limitada, pero } \\
\text { funciona en las computadoras que están en los salones. } \\
\text { La UCLA presta a los docentes... } \\
\text { Extracto del Versionante 2: }\end{array}$ & $\begin{array}{c}\text { Déficit } \\
\text { Presupuestario }\end{array}$ \\
\hline $\begin{array}{l}\text {... y comunicación como apoyo en la actividad docente } \\
\text { se debe enfrentar y superar una serie de obstáculos, } \\
\text { entre los que destacan Las Limitaciones } \\
\text { presupuestarias, esta situación impide la adquisición de } \\
\text { equipos y software de calidad, además de realizar } \\
\text { inversiones en infraestructura de redes.... } \\
\text { Extracto del Versionante 3: }\end{array}$ & $\begin{array}{c}\text { Déficit } \\
\text { Presupuestario }\end{array}$ \\
\hline $\begin{array}{l}\text {...problemas como los siguientes: existe una dotación } \\
\text { insuficiente de herramientas tecnológicas en las aulas de } \\
\text { clase, motivado a que actualmente, la mayoría de las } \\
\text { aulas de clase carecen de equipos de computación, } \\
\text { video beam y conexión a Internet. Igualmente, esta } \\
\text { situación de insuficiencia se... } \\
\text { Interrogante 2: ¿Qué herramientas didácticas utilizas } \\
\text { para desarrollar tus actividades de aula? } \\
\text { Extracto del Versionante } 1 \text {. }\end{array}$ & $\begin{array}{c}\text { Déficit } \\
\text { Presupuestario }\end{array}$ \\
\hline $\begin{array}{l}\text {...digital; cuando la sección es ubicada en salones donde } \\
\text { se tiene acceso a internet y hay disponibilidad de } \\
\text { computadora y video beam, entonces, la clase se hace } \\
\text { más dinámica y efectiva; el problema es que no todos los } \\
\text { salones cuentan con esa tecnología y debemos volver a } \\
\text { la pizarra y los marcadores... } \\
\text { Extracto del Versionante 2: }\end{array}$ & TIC's \\
\hline $\begin{array}{l}\text {...de estos recursos, existen diversidad de herramientas, } \\
\text { pero me gustan más las que se usan con las } \\
\text { computadoras como es el caso del correo electrónico, el }\end{array}$ & TIC's \\
\hline
\end{tabular}
cual permite mantener un intercambio de información con 


\section{Texto}

Categoría

el grupo....

Extracto del Versionante 3:

...además de estos, sería más productivo si en los

TIC's salones de clase se tuviesen disponibles a toda hora computadora, videoproyector, accesorios de sonido (cornetas), memoria USB, software Excel para realizar cálculos y registros contables; software Microsoft PowerPoint, para realizar presentaciones...

Fuente: La Autora (2018).

Figura nro. 1. Hallazgos y Resultados.
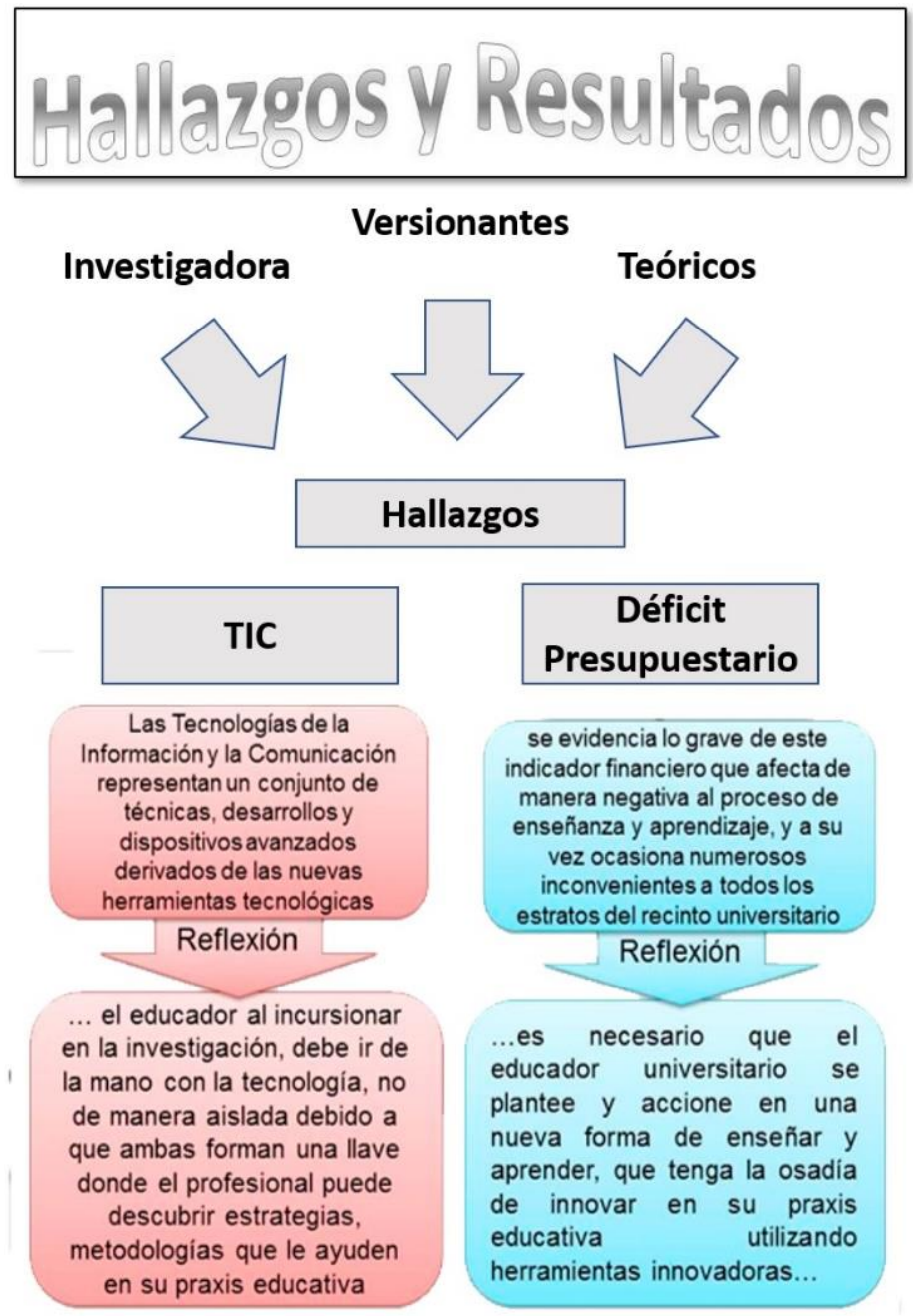

Fuente: La Autora (2018). 


\section{Reflexiones Conclusivas}

Luego de haber realizado el recorrido epistémico sobre todos los elementos que coadyuvaron a expresar una visión cercana sobre La Transcomplejidad como Agente Reflexivo de la Investigación en la Integración de las Tecnologías de Información y Comunicación en el Proceso de Enseñanza y Aprendizaje de los Docentes, en el Decanato de Ciencias y Tecnologías de la (UCLA), afloraron en mí, reflexiones más profundas en cuanto a:

Desde mi sentir docente considero que, a pesar del déficit presupuestario al que atraviesa esta casa de estudios, es necesario que el educador universitario se plantee y accione en una nueva forma de enseñar y aprender, que tenga la osadía de innovar en su praxis educativa utilizando herramientas innovadoras que posean los estudiantes, como por ejemplo celulares inteligentes, las redes sociales y la internet, pero todo ello bajo una constante actualización que le permitan incorporarlas con seguridad y destreza en su praxis tanto del saber formal como del saber cotidiano.

Como docente universitario en ejercicio, considero que el educador debe ir más allá de ser un simple facilitador, un dador de clases; para ello debe avocarse a la investigación ya que de esta manera lograría adquirir nuevos conocimientos, para esto debe apropiarse de habilidades y destrezas en el manejo de la tecnologías de la información y la comunicación, y así poder indagar y descubrir un bagaje de estrategias que les permita ser aplicada en su praxis educativa, dándole otra dimensión y concepción en la forma de como el docente universitario enseña y como el estudiante aprende.

A manera conclusiva, exhorto a la masa docente de las diferentes universidades del mundo, a que mantenga una condición abierta, innovadora y creativa, de cara a los procesos investigativos, volcados al trabajo productivo y participativo en equipos, siendo proactivos, para así afrontar cualquier reto educativo que este por venir. 


\section{Referencias}

Albornoz, O. (2006). La Universidad Latinoamericana entre Davos y Porto

Alegre. Caracas, Venezuela: Editorial CEA, S.A., pág. 28.

DRAE (2017). Universidad. Diccionario de la Lengua Española. 23. a ed. Madrid: España: Real Academia Española, párr. 1.

Gadamer, H. (1996). Verdad y Método. Fundamentos de una Hermenéutica

Filosófica. Salamanca, España: Editorial Sígueme, pág. 23.

González, J. (2012). Teoría Educativa Transcompleja. Educación Compleja y Transdisciplinar. Primera Edición, ISBN: 978-99954-0-697-4. La Paz, Bolivia: Instituto Internacional de Integración Convenio Andrés Bello,

pág. 8. Recuperado de: https://es.scribd.com/document/332841680/01pdf-transcomplejidad-pdf

González, R. (2007). Los Procesos de Construcción del Conocimiento desde una visión compleja. México D.F.: Editores McGrawHill/Interamericana, pág. 17.

Habermas, J. (1986). Teoría de la acción comunicativa. Editorial: Taurus. Ley de Universidades (1970). Artículo 1. Palacio de Miraflores. Caracas,

Venezuela: El Consejo Universitario, pág. 1. Recuperado de: https://www.google.co.ve/url?sa=t\&rct=j\&q=\&esrc=s\&source=web\&cd= 1\&ved=2ahUKEwih5J Dp8rcAhXP1IMKHbCtDE4QFjAAegQIABAC\&ur I=https\%3A\%2F\%2Fwww.oei.es\%2Fhistorico\%2Fquipu\%2Fvenezuela \%2FLey de universidades.pdf\&usg=AOvVaw1-

Qba3ER38iVdbRoYyU LW

Martínez, M. (2004). Ciencia y Arte en la Metodología Cualitativa. México D.F.: Editorial Trillas, pág. 139.

Molina, E. (2018). Programa de Reforestación como Alternativa Ambiental en la Recuperación de Espacios de Recreación y Esparcimiento. Revista Scientific, 3(7), 121-139. Recuperado de:

https://doi.org/10.29394/Scientific.issn.2542-2987.2018.3.7.6.121-139 
Morales, M. (2014a,b,c,d). Percepción del Profesorado y del Alumnado de la Facultad de Ciencias de la Educación de la Universidad de Granada Acerca de la Utilización de las Tic por Parte del Profesorado Universitario y de su Integración en el Proceso de Enseñanza-Aprendizaje. Tesis Doctoral, ISBN 978-84-8439-962-9. España: Universidad de Jaén, págs. 1, 199, 233, 433. Recuperado de: https://www.google.co.ve/url?sa=t\&rct=i\&q=\&esrc=s\&source=web\&cd= 2\&ved=2ahUKEwj3rdniqczcAhXss1kKHZOfB58QFjABegQIABAC\&url= http\%3A\%2F\%2Fruja.ujaen.es\%2Fbitstream\%2F10953\%2F689\%2F1 \%2F9788484399629.pdf\&usg=AOvVaw2lbEQv9uBVFOi9j 16hp8Z

Morín, E. (2010). La Mente Bien Ordenada. España: Editorial Seix Barral, pág. 22.

Sandín, M. (2003). Investigación Cualitativa en Educación. España: Editorial Mc Graw and Hill, pág. 123.

Sosa, O. (2013). Impacto de la Cultura de las Comunicaciones y sus Tecnologías en las Reconfiguraciones Organizacionales. Revista Arbitrada del Centro de Investigación y Estudios Gerenciales A.C., 3(3), 13-30, ISSN: 2244-8330. Recuperado de:

https://docplayer.es/79400926-Impacto-de-la-cultura-de-lascomunicaciones-y-sus-tecnologias-en-las-reconfiguracionesorganizacionales.html

Taylor, S., \& Bogdan, R. (2002). Introducción a los Métodos Cualitativos de Investigación: la búsqueda de los significados. Barcelona, Madrid, España: Paidós, pág. 20.

Villegas, C., \& Schavino, N. (2012). La Transcomplejidad: Una Nueva Visión del Conocimiento. Segunda Edición. San Juan de los Morros, Venezuela: Editorial Redit, pág. 55. 


\section{Mayibe Josefina Agüero Rodríguez \\ e-mail: mayibe.j.aguero@gmail.com}

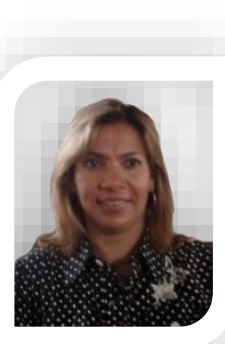

Nacida en la ciudad de Barquisimeto, estado Lara, Venezuela. Profesional Universitario en el área de Informática, Ingeniero (UCLA), Doctora en Ciencia de la Educación (UFT), Profesora en Informática (UPEL), Magister en Gerencia Empresarial (UFT) y Magister en Tecnología Educativa (UNEFA). He participado como Tutor, Asesor y Jurado de Trabajos de pre y post grado en la UPEL, UNEFA, UCLA, UPTAEB; Arbitro de Ensayos y Artículos Científicos, experta en procesos E-learning FATLA, UNEFA, UFT y UCLA; Terapeuta en PNL; experiencia en el uso de estrategias didácticas en la praxis educativa, habilidades y destrezas en el área de la comunicación efectiva, resolución de conflictos, ética laboral y talleres motivacionales.

El contenido de este manuscrito se difunde bajo una Licencia de Creative Commons ReconocimientoNoComercial-Compartirlgual 4.0 Internacional 